TIFR/TH/97-12

UCRHEP-T186

(revised)

\title{
New interactions in neutrino oscillations with three light flavors
}

\author{
Ernest Ma用 \\ Physics Department \\ University of California, Riverside \\ CA 92521. \\ Probir Roy \\ Tata Institute of Fundamental Research \\ Mumbai 400 005, India.
}

September 1997

\begin{abstract}
If one assumes solar and LSND neutrino oscillations to explain the corresponding data, then the atmospheric neutrino deficit cannot be accommodated within the Standard Model with three light flavors, unless one ignores the data's zenith-angle dependence. We propose a novel solution to this problem by postulating large anomalous diagonal $\nu_{\tau}$-quark interactions which affect $\nu_{\mu}-\nu_{\tau}$ oscillations traversing the Earth and induce the observed zenith-angle dependence.
\end{abstract}

*email: ernestma@ucrac1.ucr.edu

${ }^{\dagger}$ email: probir@theory.tifr.res.in 
Three flavors of massless lefthanded weakly interacting neutrinos occur in the Standard Model. Experimental studies at the Large Electron Positron (LEP) collider have definitively established the number of light weak neutrino flavors to be three. However, the masslessness of any neutrino is not predicted on fundamental grounds. Beyond the Standard Model, theoretical arguments exist showing how neutrinos could acquire tiny Majorana [1] or Dirac [2] masses. On the experimental front, there is indirect evidence of small nonvanishing neutrino masses from three different kinds of phenomena pertaining to neutrino oscillations. (1) The observed depletion [3] of the solar neutrino flux from the prediction of the standard solar model in different segments of the solar neutrino energy spectrum, (2) the claimed discovery [4] of $\bar{\nu}_{\mu}-\bar{\nu}_{e}$ and $\nu_{\mu}-\nu_{e}$ oscillations by the Liquid Scintillator Neutrino Detector (LSND) experiment and (3) the deficit [5] in the atmospheric neutrino flux, measured on the ground in terms of the ratio of ratios $R \equiv\left(\nu_{\mu}+\bar{\nu}_{\mu}\right):\left(\nu_{e}+\bar{\nu}_{e}\right)_{\text {expt. }} /\left(\nu_{\mu}+\bar{\nu}_{\mu}\right):\left(\nu_{e}+\bar{\nu}_{e}\right)_{M C}$, $M C$ standing for the Monte-Carlo expectation - all point to nonzero neutrino masses. The canonical best fits to the data from the above three different experimental studies cannot be accommodated within the hypothesis of only three light neutrino flavors because of the three nonoverlapping ranges of $\delta m^{2}$ involved. This and other considerations from astrophysics and cosmology have led to the speculation of the existence [6] of a fourth sterile (i.e. electroweak singlet) light neutrino as a possible way of reconciling all of the known data. On the other hand, if only three neutrino flavors are assumed, two of the three possible neutrino oscillations can be explained and the question is to what extent the third can be accommodated. Previous studies have chosen the exception to be either the atmospheric data [7] or the solar data [8]. A recent general analysis [9] shows that the former hypothesis is in fact favored.

It seems to us that the results of the solar neutrino and the LSND experiments are quite unambiguous, assuming the absence of unknown sources of systematic error. In contrast, the detailed conclusions from the atmospheric neutrino experiment seem to depend sensitively 
on the intricacies of the Monte-Carlo simulations [5] used. It may, therefore, be more profitable to consider three-flavor scenarios which naturally explain the solar neutrino and the LSND data and then to explore the observed atmospheric neutrino anomaly within those. That leads one naturally to the scheme of Ref. [7]. This scheme, in which the interactions are exclusively those of the Standard Model, leads to a universal value of $R$ that can give acceptable fits to the sub-GeV and multi-GeV data, integrating over all zenith angles. However, it disallows any measurable dependence of the data on the zenith angle predicting an essentially flat distribution.

As in Ref. [7], we too start with only three light neutrino flavors and Standard Model interactions, but then we extend the latter to include the possibility of anomalous neutrino interactions [10]. Specifically, we allow $\nu_{\tau}$ to have any large nonstandard diagonal fourfermion (effectively contact) interaction with quarks. This is motivated by two facts: (1) the reported observation [11] of anomalous $e^{+}$-quark interactions at HERA, which suggests the possibility of anomalous lepton-quark interactions in general; (2) among the three known neutrinos, $\nu_{\mu, e}$ are strongly forbidden by experimental constraints to have such large interactions while there exist essentially no restrictions on $\nu_{\tau}$. (In particular, there could be a heavy vector boson coupling only to leptons of the third generation but to the light quarks as well). As we show below, this will result in the novel possibility of an induced zenith-angle dependence for atmospheric neutrino oscillations, which appears to be favored by the data.

With an anomalous $\nu_{\tau}$-quark interaction, the survival probability $R$ may vary with the zenith angle in atmospheric neutrino oscillations despite a large $\delta m^{2}$ chosen at around $0 . .25$ $\mathrm{eV}^{2}$ to satisfy the LSND data. Since the interaction cross section (in the detector) of neutrinos is roughly 3 times that of antineutrinos, and the neutrino flux is somewhat larger than the antineutrino flux [12] at higher energies, such a variation is potentially able to explain the multi-GeV atmospheric data. The extra $\nu_{\tau}$ interactions inside the sun are offset by a 
$\delta m^{2}$ much larger than it would be for the canonical matter-enhanced effect, as explained below.

We start with the following approximate mass eigenstates: $\nu_{1} \sim \nu_{e}, \nu_{2} \sim c_{0} \nu_{\mu}+s_{0} \nu_{\tau}$, $\nu_{3} \sim-s_{0} \nu_{\mu}+c_{0} \nu_{\tau}$, where $c_{0} \equiv \cos \theta_{0}, s_{0} \equiv \sin \theta_{0}$, and $\theta_{0}$ is not small. We choose $m_{1} \sim 0$, $m_{2} \sim 10^{-2} \mathrm{eV}$, and $m_{3} \sim 0.5 \mathrm{eV}$. We then allow $\nu_{1}$ to mix with $\nu_{3}$ with a small angle $\theta^{\prime}$ and the new $\nu_{1}$ to mix with $\nu_{2}$ with a small angle $\theta$. The exact mass eigenstates are then

$$
\begin{aligned}
& \nu_{1}=c c^{\prime} \nu_{e}+c s^{\prime}\left(-s_{0} \nu_{\mu}+c_{0} \nu_{\tau}\right)-s\left(c_{0} \nu_{\mu}+s_{0} \nu_{\tau}\right), \\
& \nu_{2}=c\left(c_{0} \nu_{\mu}+s_{0} \nu_{\tau}\right)+s c^{\prime} \nu_{e}+s s^{\prime}\left(-s_{0} \nu_{\mu}+c_{0} \nu_{\tau}\right), \\
& \nu_{3}=c^{\prime}\left(-s_{0} \nu_{\mu}+c_{0} \nu_{\tau}\right)-s^{\prime} \nu_{e} .
\end{aligned}
$$

Let us now turn one-by-one to the three sets of neutrino oscillation data.

LSND: $\quad \bar{\nu}_{\mu}-\bar{\nu}_{e}$ oscillations, as probed in this experiment, are controlled by $\delta m_{31}^{2} \sim 0.25$ $\mathrm{eV}^{2}$. Any significantly higher value chosen for $\delta m_{31}^{2}$ will be in contradiction with restrictions imposed by the search for $\nu_{\mu}$ disappearance in the CDHS experiment [13] for large angles (which will be needed later in explaining the atmospheric neutrino effect). On the other hand, for such a value of $\delta m^{2}$, the LSND data [4] imply a mixing angle $\chi$ with $\sin 2 \chi \simeq 0.19$. These numbers are just about compatible with the constraints of the Bugey experiment [14]. Comparing with (3), we find $2 s_{0} s^{\prime} c^{\prime} \simeq 0.19$.

Solar neutrino data: The canonical solution for solar neutrino oscillations takes $\nu_{1} \sim \nu_{e}$ and $\nu_{2} \sim$ a linear combination of $\nu_{\mu}$ and $\nu_{\tau}$, with $m_{2}>m_{1}$ and some mixing between $\nu_{1}$ and $\nu_{2}$. In its passage through the sun, $\nu_{e}$ gets an extra induced mass because of its forward scattering with the electrons. The matching of this mass with $\delta m_{21}^{2}$ produces the well-known MSW effect [15]. Here we have new extra diagonal $\nu_{\tau}$-quark interactions. Consequently, a larger $\delta m_{21}^{2}$ is needed to cancel against the induced $\nu_{\tau}$ mass, which should be negative in this case [16]. 
Let us examine in detail the effect of $\nu_{\tau}$-quark interactions on the passage of electron neutrinos through the sun. We write this new interaction as [10]

$$
\mathcal{L}_{\text {new }}=-\sqrt{2} \bar{\nu}_{\tau L} \gamma_{\mu} \nu_{\tau L}\left(G_{\tau \tau V}^{q} \bar{q} \gamma^{\mu} q+G_{\tau \tau A}^{q} \bar{q} \gamma^{\mu} \gamma_{5} q\right)
$$

for all quarks $q$. Note that the $\tau$ neutrino and the $\tau$ antineutrino are known from $\tau$-decay properties to be lefthanded and righthanded respectively. Therefore, all possible four-fermion interactions involving them and quarks can be brought into the form (4) by Fierz transformations. Only the vector coupling $G_{\tau \tau V}^{q}$ contributes to the potential relevant to forward scattering while its contributions for the neutrino and antineutrino cases are equal in magnitude but opposite in sign. We define $\epsilon_{q}^{\prime} \equiv G_{\tau \tau V}^{q} / G_{F}$ as in Ref. [10]. Note that we do not require any flavor-changing interactions which would have been necessary to obtain oscillations if $\delta m^{2}=0$.

Solar neutrino oscillations occur between $\nu_{e}$ and $\nu_{\alpha}=c_{0} \nu_{\mu}+s_{0} \nu_{\tau}$ with angle $\theta$. Since $m_{3}^{2} \gg m_{2}^{2}, \nu_{\beta}=-s_{0} \nu_{\mu}+c_{0} \nu_{\tau}$ effectively decouples. Hence the relevant evolution equation can be written, after rotating away a common phase, as [10]

$$
4 i E_{\nu} \frac{d}{d t}\left(\begin{array}{c}
\nu_{e} \\
\nu_{\alpha}
\end{array}\right) \simeq\left(\begin{array}{cc}
0 & m_{2}^{2} \sin 2 \theta \\
m_{2}^{2} \sin 2 \theta & 2 m_{2}^{2} \cos 2 \theta+4 \sqrt{2} G_{F} E_{\nu}\left(s_{0}^{2} \epsilon_{q}^{\prime} N_{q}-N_{e}\right)
\end{array}\right)\left(\begin{array}{c}
\nu_{e} \\
\nu_{\alpha}
\end{array}\right) .
$$

In (5), $N_{q} \epsilon_{q}^{\prime} \equiv N_{u} \epsilon_{u}^{\prime}+N_{d} \epsilon_{d}^{\prime}$ and $N_{e, u, d}$ is the number of (electrons, $u$-quarks, $d$-quarks) per unit solar volume. The coefficient $s_{0}^{2} \equiv \sin ^{2} \theta_{0}$ of the $\epsilon_{q}^{\prime} N_{q}$ term in the second diagonal matrix element originates from the $3 \times 3 \rightarrow 2 \times 2$ flavor matrix reduction. In order to have a large $\epsilon_{q}^{\prime}$ and yet satisfy the resonance condition for solar-neutrino flavor conversion, we see that $m_{2}$ should be larger than its canonical value of $2.45 \times 10^{-3} \mathrm{eV}$, and $\epsilon_{q}^{\prime}$ should be negative. [If $\epsilon_{q}^{\prime}$ comes from $R$-parity violating squark exchange, then it must be positive; but if it comes from vector exchange, then it may be of either sign.]

We now assume as a crude approximation that $N_{q} \simeq 4 N_{e}$ in the sun. It then follows from 
(5) that the effective mixing angle for $\nu_{e}-\nu_{\alpha}$ oscillations in solar matter is given by

$$
\tan 2 \theta_{m}^{S}=\frac{\sin 2 \theta}{\cos 2 \theta+2 \sqrt{2} G_{F} m_{2}^{-2} E_{\nu}\left(4 s_{0}^{2} \epsilon_{q}^{\prime}-1\right) N_{e}}
$$

and the MSW resonance condition [15] is

$$
m_{2}^{-2}\left(-4 s_{0}^{2} \epsilon_{q}^{\prime}+1\right)=\cos 2 \theta\left(2 \sqrt{2} G_{F} N_{e} E_{\nu}\right)^{-1}
$$

In the canonical MSW solution, the left-hand side is $\left(6 \times 10^{-6} \mathrm{eV}^{2}\right)^{-1}$. It can thus be matched with the requirement

$$
s_{0}^{2} \epsilon_{q}^{\prime} \simeq-3.92=-4.17\left(m_{2}^{2} / 10^{-4} \mathrm{eV}^{2}\right)+0.25
$$

Our seemingly arbitrary choice of $\delta m_{21}^{2} \sim 10^{-4} \mathrm{eV}^{2}$ is now seen as a reasonable value so that $\epsilon_{q}^{\prime}$ can be large enough to be relevant for the following discussion on the atmospheric neutrino data. Note that the same range of $\theta$, i.e. near 0.04 , works here as well as in the standard MSW solution for solar neutrino oscillations.

Atmospheric neutrino data: The depletion in the flux of muon neutrinos and antineutrinos, produced in the earth's upper atmosphere, is caused by $\nu_{\mu}-\nu_{\tau}$ flavor oscillations which occur between the physical states $\nu_{2}$ and $\nu_{3}$ with $\delta m_{32}^{2} \sim 0.25 \mathrm{eV}^{2}$ and angle $\theta_{0}$. The oscillation wavelength "in vacuo" is $\lambda \sim 4 \pi E_{\nu} / \delta m_{32}^{2} \sim 10\left(E_{\nu} / \mathrm{GeV}\right) \mathrm{km}, E_{\nu}$ being the neutrino energy. Hence, for $E_{\nu}<10 \mathrm{GeV}$, several oscillations occur in the earth's atmosphere. Consequently, one obtains the classical survival probability $P_{0}=c_{0}^{4}+s_{0}^{4}=1-\frac{1}{2} \sin ^{2} 2 \theta_{0}$. The choice of $s_{0} \simeq 0.47$ yields $R=P_{0} \simeq 0.66$ for the ratio of ratios, in reasonable agreement with the data for downward going neutrinos and antineutrinos. This implies $s^{\prime} \simeq 0.21$.

For neutrinos and antineutrinos coming downward, the density of the atmosphere is negligible for the new diagonal $\nu_{\tau}$-quark interactions to be of any importance. For upward moving ones, the density of the earth turns out to be in the right range for them to make a difference. Since $\nu_{e}$ gets effectively decoupled from the $\nu_{\mu}-\nu_{\tau}$ oscillation problem, we now 
have

$$
4 i E_{\nu} \frac{d}{d t}\left(\begin{array}{c}
\nu_{\mu} \\
\nu_{\tau}
\end{array}\right) \simeq\left(\begin{array}{cc}
0 & m_{3}^{2} \sin 2 \theta_{0} \\
m_{3}^{2} \sin 2 \theta_{0} & 2 m_{3}^{2} \cos 2 \theta_{0}+4 \sqrt{2} G_{F} E_{\nu} \epsilon_{q}^{\prime} N_{q}
\end{array}\right)\left(\begin{array}{c}
\nu_{\mu} \\
\nu_{\tau}
\end{array}\right) .
$$

For the earth we estimate an average $N_{q} \sim 9 \times 10^{30} \mathrm{~m}^{-3}$. Thus if one chooses to define the parameter $X \equiv \epsilon_{q}^{\prime} E_{\nu} /(10 \mathrm{GeV})$, the effective mixing angles in terrestrial matter (a) $\theta_{m}^{E}$ between $\nu_{\mu}$ and $\nu_{\tau}$ and (b) $\bar{\theta}_{m}^{E}$ between $\bar{\nu}_{\mu}$ and $\bar{\nu}_{\tau}$ are respectively given by

$$
\tan 2 \theta_{m}^{E}=\frac{\sin 2 \theta_{0}}{\cos 2 \theta_{0}+0.091 X}, \quad \tan 2 \bar{\theta}_{m}^{E}=\frac{\sin 2 \theta_{0}}{\cos 2 \theta_{0}-0.091 X}
$$

with $\cos 2 \theta_{0}=\mp 0.091 X$ as the resonance conditons.

For sub-GeV neutrinos, the $X$ term is insignificant, but for multi-GeV neutrinos it may become large enough for the resonance condition to be satisfied. Assuming adiabaticity, the neutrino and antineutrino flavor survival probabilities are described well by the formulae [17]

$$
\begin{aligned}
P\left(\nu_{\mu} \rightarrow \nu_{\mu}\right) & =\frac{1}{2}\left(1+\cos 2 \theta_{0} \cos 2 \theta_{m}^{E}\right), \\
\bar{P}\left(\bar{\nu}_{\mu} \rightarrow \bar{\nu}_{\mu}\right) & =\frac{1}{2}\left(1+\cos 2 \theta_{0} \cos 2 \bar{\theta}_{m}^{E}\right),
\end{aligned}
$$

where $\cos 2 \theta_{m}^{E}$ and $\cos 2 \bar{\theta}_{m}^{E}$ are computed from (11). Although the conditions for adiabaticity may not be satisfied, our purpose is to try to find the maximum effect for a given magnitude of the anomalous interaction which is of course unknown. Any nonadiabaticity would only tend to diminish this effect.

Owing to the opposite signs of the media contributions to neutrino and antineutrino oscillations, matter effects will get somewhat diluted. However, there are two important factors to be considered. First, the initial $\nu_{\mu}$ flux is larger than the $\bar{\nu}_{\mu}$ flux for multi-GeV neutrinos. In the upper atmosphere, $\nu_{\mu}\left(\bar{\nu}_{\mu}\right)$ is produced together with $\mu^{+}\left(\mu^{-}\right)$from $\pi^{+}\left(\pi^{-}\right)$ decay. The subsequent decay of $\mu^{+}\left(\mu^{-}\right)$to $\bar{\nu}_{\mu}\left(\nu_{\mu}\right)$ will equalize the total number of $\nu_{\mu}$ and $\bar{\nu}_{\mu}$, but there is an energy dependence and given that the $\mu^{+} / \mu^{-}$ratio is larger than one [12], we allow a factor of $r=$ ratio of the $\nu_{\mu}$ to $\bar{\nu}_{\mu}$ flux in our following discussion. Second, in the 
detection of atmospheric neutrinos, there is no experimental measurement of the charge of the resulting leptons. Specifically, $\mu$-like events include both $\mu^{-}$and $\mu^{+}$, but they are not separated. Now $\sigma_{\nu} \simeq 3 \sigma_{\bar{\nu}}$ for an isoscalar target, hence the measured probability is weighted:

$$
P_{m} \simeq \frac{3 r P+\bar{P}}{3 r+1}
$$

In the absence of media effects, $P=\bar{P}$ even with oscillations (assuming CP conservation), hence $P_{m}=P=\bar{P}$. In the presence of media effects, $P \neq \bar{P}$, so the above expression for $P_{m}$ should be used.

Suppose we now make the same choice of $s_{0} \simeq 0.47$ which leads to a suppression probability $P_{0}=0.66$ for the downward travelling neutrinos. Then, for an optimistically large and negative value of $X=-15$, we get $P=0.31$ and $\bar{P}=0.76$, hence $P_{m}$ is lowered to 0.39 if $r=1.5$ in Eq. (14) or 0.42 if $r=1.0$. This is a potential explanation of the observation of a smaller $R$ for atmospheric neutrinos going through the earth (zenith angle $>\pi / 2$ ) where $R=P_{m}$ than for those coming down through only the atmosphere (zenith angle $<\pi / 2$ ) where $R=P_{0}$.

There have been studies [18] of $R$-parity violating squark interactions, scalar and vector leptoquarks, as well as contact interactions of neutrinos. Whereas these may be related to our proposed effective interaction, they are all restricted to be small. We consider instead a vector boson $B$ which couples to $\bar{u} \gamma^{\mu} u+\bar{d} \gamma^{\mu} d-\bar{\nu}_{\tau} \gamma^{\mu} \nu_{\tau}-\bar{\tau}_{L} \gamma^{\mu} \tau_{L}$ with coupling $g_{B}$. This interaction would result in a negative $X$ as desired. Using the result of a previous model [19] where $B$ couples to baryon number, and allowing for the fact that here $B$ couples to $u$ and $d$ but not $s, c$, and $b$, we find $\alpha_{B} \equiv g_{B}^{2} / 4 \pi \leq 0.057$. We now require $g_{B}^{2} / m_{B}^{2}=15 G_{F}$, hence $m_{B}=64 \mathrm{GeV}$ is allowed. The deviation from $e-\mu-\tau$ universality in $Z$ decay is then given by

$$
\frac{\alpha_{B}}{2 \pi}\left(\frac{g_{L}^{2}}{g_{L}^{2}+g_{R}^{2}}\right)_{\tau}\left(F_{1}+F_{2}\right)=\frac{0.057}{2 \pi}(0.5735)(1.32)=0.0069
$$


where $F_{1}$ and $F_{2}$ are well-known functions [19] of the ratio $m_{B}^{2} / M_{Z}^{2}$ which is about 0.5 for $m_{B}=64 \mathrm{GeV}$. The above is exactly two standard deviations from the experimental data [20], taking into account the kinematical correction due to $m_{\tau}$ in $Z \rightarrow \tau^{+} \tau^{-}$. The deviation in the total invisible width of $Z$ is 0.012 versus the standard-model value of 3 , which is again two standard deviations from the data [20]. This is then a possible explicit model for our scenario.

Our scenario will have the following consequences for the forthcoming experiments. Solar neutrino experiments will confirm the MSW solution, but the interpretation of $\delta m^{2}$ is subject to the ambiguity that it could be $\delta m^{2} /\left(1-4 s_{0}^{2} \epsilon_{q}^{\prime}\right)$ instead. However, the anomalous $\nu_{\tau}$-quark interactions will be observable at SNO, thereby resolving this ambiguity. Both $\nu_{\mu} \rightarrow \nu_{e}$ and $\nu_{\mu} \rightarrow \nu_{\tau}$ conversion experiments will measure a $\delta m^{2}$ at around $0.25 \mathrm{eV}^{2}$, but $\sin ^{2} 2 \theta_{\mu, e} \sim 0.036$ while $\sin ^{2} 2 \theta_{\mu, \tau} \sim 0.69$. The former is outside the region being probed by reactor experiments ( $\bar{\nu}_{e}$ disappearance) such as Chooz and Palo Verde; the latter is outside that being probed by short-baseline accelerator experiments $\left(\nu_{\tau}\right.$ appearance) such as CHORUS and NOMAD. On the other hand, both regions are covered by all the proposed long-baseline experiments (either through $\nu_{\mu}$ disappearance, or $\nu_{e}$ and $\nu_{\tau}$ appearances) such as MINOS, K2K (KEKPS/Super-Kamiokande) and CERN-SPS/ICARUS.

More immediately, the new data from Super-Kamiokande, Soudan 2, and MACRO on $\nu_{\mu}+\bar{\nu}_{\mu}$ events through the earth will be sensitive to the anomalous $\nu_{\tau}$-quark interactions. There should be an energy dependence as well as a zenith-angle dependence. In particular, the zenith-angle dependence should be absent or much smaller for sub-GeV data. For a zenith angle near zero, our proposal is easily distinguishable from the $\delta m^{2} \sim 10^{-2} \mathrm{eV}^{2}$ oscillation interpretation because we have $R=P_{0}$ whereas the latter would require $R \sim 1$, owing to the short distance between production and detection in that case. To test our hypothesis further, the detection and acceptance efficiencies of neutrinos versus antineutrinos have to be 
understood in more detail. Better yet, the capability of these experiments for distinguishing neutrinos from antineutrinos should be explored [21].

\section{Acknowledgements}

P.R. wishes to acknowledge the hospitality of the Department of Physics, UC Riverside, during the course of this work. This work was supported in part by the U. S. Department of Energy under Grant No. DE-FG03-94ER40837. 


\section{REFERENCES}

[1] M. Gell-Mann, P. Ramond and R. Slansky, in Supergravity (ed. P. Van Nieuwenhuizen and D.Z. Freedman, North-Holland, 1979), p315. T. Yanagida, in Proceedings of the Workshop on the Unified Theory and the Baryon Number in the Universe (ed. O. Sawada and A. Sugamoto, KEK Report No. 79-18, Tsukuba, Japan, 1979).

[2] P. Roy and O. Shanker, Phys. Rev. Lett. 52, 713 (1984); Phys. Rev. D30, 1949 (1984); M. Roncadelli and D. Wyler, Phys. Lett. 133B, 325 (1983).

[3] For reviews of the subject and many references see the following articles: R.S. Raghavan, Science 267, 45 (1995). J.H. Bahcall and M.H. Pinsonneault, Rev. Mod. Phys. 67, 1 (1995). R. Davis, Prog. Part. Nucl. Phys. 32, 13 (1994). Y. Fukuda et al., Phys. Rev. Lett. 77, 1683 (1996). P. Anselmann et al., Phys. Lett. B327, 377 (1994); 342, 440 (1995). J.N. Abdurashitov et al., Phys. Lett. B328, 234 (1994).

[4] C. Athanassopoulos et al., Phys. Rev. Lett. 75, 2650 (1995); 77, 3082 (1996). The high $\delta m^{2}$ region, allowed by this experiment, has recently been ruled out by the CCFR group: A. Romosan et al., Phys. Rev. Lett. 78, 2912 (1997). The observation of $\nu_{\mu} \leftrightarrow \nu_{e}$ oscillations has been recently claimed by the LSND group: C. Athanassopoulos et al., nucl-ex/9706006.

[5] Y. Fukuda et al., Phys. Lett. B335, 237 (1994) and earlier references therein. R. Becker-Szendy et al., Phys. Rev. D46, 3720 (1992). D. Casper et al., Phys. Rev. Lett. 66, 2561 (1991).

[6] J.R. Primack, J. Holtzman, A. Klypin and D.O. Caldwell, Phys. Rev. Lett. 74, 2160 (1995). G.M. Fuller, J.R. Primack, and Y.-Z. Qian, Phys. Rev. D52, 1288 (1995).. 
E. Ma and P. Roy, Phys. Rev. D52, R4780 (1995). See also D.O. Caldwell and R.N. Mohapatra, Phys. Rev. D48, 3259 (1993); J.T. Peltoniemi and J.W.F. Valle, Nucl. Phys. B406, 409 (1993).

[7] C. Y. Cardall and G. M. Fuller, Phys. Rev. D53, 4421 (1996). See also the earlier work of S. M. Bilenky, A. Bottino, C. Giunti, and C. W. Kim, Phys. Lett. B356, 273 (1995); K. S. Babu, J. C. Pati, and F. Wilczek, ibid., B359, 351 (1995); G. L. Fogli, E. Lisi, and G. Scioscia, Phys. Rev. D52, 5334 (1995).

[8] A. Acker and S. Pakvasa, Phys. Lett. B397, 209 (1997). However, the prediction of energy independence here is disfavored by the data: P.I. Krastev and S.T. Petcov, Phys. Lett. B395, 69 (1997).

[9] G.L. Fogli, E. Lisi, D. Montanino, and G. Scioscia, Phys. Rev. D56, 4365 (1997).

[10] M.M. Guzzo, A. Masiero, and S.T. Petcov, Phys. Lett. B260, 154 (1991); E. Roulet, Phys. Rev. D44, R935 (1991); V. Barger, R.J.N. Phillips, and K. Whisnant, Phys. Rev. D44, 1629 (1991); P.I. Krastev and J.N. Bahcall, hep-ph/9703267.

[11] H1 Collaboration, C. Adloff et al., Z. Phys. C74, 191 (1997); ZEUS Collaboration, J. Breitweg et al., Z. Phys. C74, 207 (1977).

[12] Preliminary results of the HEAT and MASS cosmic ray experiments on the ratio $\mu^{+} / \mu^{-}$ are $1.64 \pm 0.08$ and $1.2 \pm 0.05$ respectively, as quoted by M. Goodman in Long Baseline Neutrino Newsletter (August 1997).

[13] F. Dydak et al., Phys. Lett. B134, 281 (1984). This $\nu_{\mu}$ disappearance experiment by itself is usually not taken to constrain the LSND data which concern only $\nu_{\mu} \rightarrow \nu_{e}$, but in our case, it is a relevant constraint because we will also consider $\nu_{\mu} \rightarrow \nu_{\tau}$ as a possible explanation of the atmospheric neutrino anomaly, using the same $\delta m^{2} \sim 0.25 \mathrm{eV}^{2}$. 
[14] Y. Declais et al., Nucl. Phys. B434, 503 (1995).

[15] L. Wolfenstein, Phys. Rev. D17, 2369 (1978). S. Mikheyev and A.Yu. Smirnov, Sov. J. Nucl. Phys. 42, 913 (1985).

[16] If it is positive, then an inverted mass hierarchy, i.e. $m_{2}<m_{1}$ would be needed.

[17] T. Kuo and J. Pantaleone, Rev. Mod. Phys. 61, 937 (1989).

[18] G. Bhattacharyya et al., Mod. Phys. Lett. A10, 1583 (1995); J. K. Mizukoshi et al., Nucl. Phys. B443, 20 (1995); O. Eboli et al., Phys. Lett. B396, 238 (1997); M. Bilenkii and A. Santamaria, Phys. Lett. B336, 91 (1994).

[19] C. D. Carone and H. Murayama, Phys. Rev. Lett. 74, 3122 (1995).

[20] P. B. Renton, Int. J. Mod. Phys. A12, 4109 (1997).

[21] For example, MACRO had considered installing magnets to measure the charges of the observed leptons, but decided against it to reduce cost. G. Giacomelli, private communication. Recently, a new experiment HANUL with such a capability has been proposed. Y. Ho, private communication. 